\title{
Ressorts Discursifs De Diffusion Des Pratiques Relevant De L'économie De Fonctionnalité
}

\author{
Rachid Abdji, Fatima Bakali \\ Université Abdelmalek Eessaadi, Tanger, Maroc
}

\begin{abstract}
Résumé : Ce papier, met en lien la théorie néo-institutionnelle et pratiques relevant de l'économie circulaire. S'appuyant sur plusieurs méthodes inductives, elle s'alimente principalement de publications de presse, de documentation institutionnelle, et d'entretiens menés avec des acteurs de tout type en prise avec l'économie circulaire. Au plan théorique, les résultats permettent de mieux comprendre le rôle des discours dans l'hybridation de pratiques, la dimension institutionnelle de l'économie circulaire, ainsi que l'institutionnalisation de pratiques qui prend appui sur des microfondations. Au plan pratique, cette thèse dévoile des ressorts discursifs de diffusion des pratiques relevant de l'économie de fonctionnalité. Elle offre des éléments de diagnostic stratégique permettant d'aborder les transformations du domaine des déchets ménagers en France. Enfin elle décrit les mécanismes de gamification qui engagent le citoyen dans le geste de tri et de réduction des déchets.
\end{abstract}

Mots-clés : Déchets Municipaux, Discours, Economie Circulaire, Economie de Fonctionnalité, Logiques Institutionnelles, Micro-Fondations des Institutions, Pratiques Hybrides.

\section{INTRODUCTION}

Dans ce papier, met en lien théorie néo-institutionnelle et pratiques relevant de l'économie circulaire. S'appuyant sur plusieurs méthodes inductives, elle s'alimente principalement de publications de presse, de documentation institutionnelle, et d'entretiens menés avec des acteurs de tout type en prise avec l'économie circulaire. Au plan théorique, les résultats permettent de mieux comprendre le rôle des discours dans l'hybridation de pratiques, la dimension institutionnelle de l'économie circulaire, ainsi que l'institutionnalisation de pratiques qui prend appui sur des micro-fondations. Au plan pratique, cette thèse dévoile des ressorts discursifs de diffusion des pratiques relevant de l'économie de fonctionnalité. Elle offre des éléments de diagnostic stratégique permettant d'aborder les transformations du domaine des déchets ménagers en France. Enfin elle décrit les mécanismes de gamification qui engagent le citoyen dans le geste de tri et de réduction des déchets.

L'analyse de la situation du système financier au regard des tendances économiques et financières, observées et attendues, a permis au Comité de dégager les constats suivants :

- Les risques macroéconomiques se sont globalement situés à un niveau modéré. En effet, le risque émanant de la position extérieure demeure limité avec des réserves internationales nettes assurant une couverture de plus de six mois d'importations de biens et services. Sur le plan intérieur, l'économie nationale a été affectée en 2016 par la contreperformance du secteur agricole et l'atonie persistante des activités non agricoles. 
Au niveau des perspectives macroéconomiques, l'évolution reste favorable en lien avec le raffermissement attendu de l'activité économique mondiale et l'accélération prévue en 2017 de la croissance nationale tirée, principalement, par le rebond du secteur agricole et la reprise progressive des activités non agricoles. Parallèlement, le déficit du compte courant et le déficit budgétaire devraient s'atténuer d'ici 2018.

- Le crédit bancaire aux entreprises non-financières a renoué avec la croissance après une contraction constatée en 2015. Le taux des créances en souffrance a, toutefois, enregistré une nouvelle hausse liée à certains secteurs en difficulté.

- Sur la base d'une étude réalisée par Bank Al-Maghrib, portant sur un échantillon de près de 14.000 entreprises non financières privées et publiques, les délais de paiement des créances inter-entreprises se sont de nouveau allongés, en particulier pour les TPME et pour certains secteurs d'activité. La mise en œuvre rapide de la réforme du cadre législatif et réglementaire relatif aux délais de paiement, finalisée au deuxième semestre 2016, devrait contribuer à l'atténuation de cette situation.

- Les bilans bancaires ont été affectés par les effets de la conjoncture économique nationale. Les banques ont ainsi connu une baisse de leurs marges d'intérêt, conjuguée à une nouvelle hausse du risque de crédit sur leurs opérations réalisées au Maroc. Leurs activités bancaires opérées à l'étranger ont, pour leur part, enregistré de bonnes performances. Au global, les banques continuent de disposer d'un bon niveau de capitalisation. Le cadre prudentiel est, par ailleurs, en cours de renforcement à l'effet de consolider la résilience du secteur.

- Le secteur des assurances continue de dégager une marge de solvabilité, en couverture du risque de souscription, largement supérieure au minimum réglementaire. Considérant, néanmoins, le passage futur vers le régime de la solvabilité basée sur les risques, les excédents de marge devraient probablement se réduire de manière significative. Par ailleurs, les plus-values latentes susceptibles d'être dégagées par les portefeuilles d'actifs de ces institutions ont connu une hausse en relation avec la performance du marché boursier.

- La réforme paramétrique du régime des pensions civiles a permis une amélioration de sa situation financière en équilibrant la tarification de ce régime pour les droits futurs de ses affiliés sans, toutefois, résorber ses engagements importants au titre des droits passés.

\section{Figure 1-Empreinte écologique vs Capacités du système Terre}

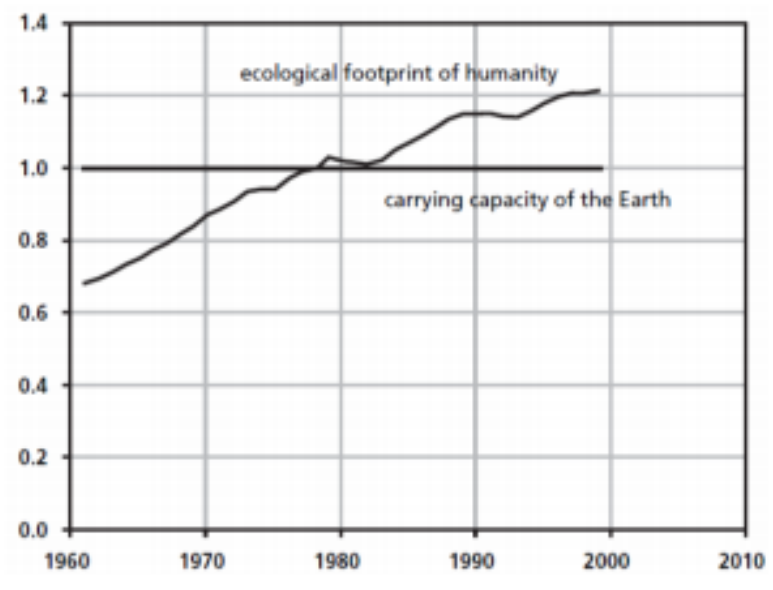


- S'agissant du marché boursier, sa liquidité s'est inscrite dans une tendance haussière. Elle reste, toutefois, insuffisante en raison essentiellement de la faiblesse du flottant. La volatilité demeure modérée et enregistre une baisse durant les quatre premiers mois de 2017 après deux années de hausses successives. En dépit d'une correction des cours en début d'année, la valorisation de la Bourse de Casablanca ressort à un niveau élevé, tirée par des investisseurs à la recherche de rentabilité dans un contexte de baisse des taux d'intérêts.

- Au niveau du marché de la dette privée, en dépit des difficultés rencontrées par quelques émetteurs, le risque de crédit reste globalement modéré. Le marché a vu l'émission d'obligations subordonnées perpétuelles d'un nouveau type (contingent convertible bonds) présentant un profil de risque différent des émissions habituelles, mais dont le volume global demeure limité. Le Comité a, par ailleurs, fait le point sur la mise en œuvre de la feuille de route relative à la contribution du secteur financier marocain à la promotion du développement durable. Il s'est également penché sur les enjeux de la finance digitale et les risques liés à la cybercriminalité.

\section{EMPREINTE ECOLOGIQUE VS CAPACITES DU SYSTEME TERRE}

La recherche en management fait état de la mise en œuvre de ces pratiques (Ghisellini et al.,2016 ; Murray et al., 2017). Pourtant, malgré les avancées considérables de la recherche et de ssuccès de mise en œuvre certains, un changement de focale est nécessaire pour opérer un passage à l'échelle sociétale. La multiplication des politiques publiques portant sur l'économie circulaire depuis les années 2010 va en ce sens. Aussi, plusieurs chercheurs ont appelé à renouer avec une approche holistique apte à rendre compte du caractère systémique de la transition vers une économie circulaire. (Ghisellini et al., 2016 ; Kirchherr et al., 2017 ; Murray et al., 2017). La présente thèse s'inscrit dans cette perspective. Elle rejoint cette idée que la diffusion des pratiques d'économie circulaire tient aussi, et peut-être surtout, à des facteurs institutionnels, au sens de la sociologie des institutions (DiMaggio \& Powell, 1983 ; Meyer \& Rowan, 1977 ; Zucker, 1977).

Les cas de mise en œuvre qui ont réussi au niveau de l'organisation ou du réseau d'organisations (ex. parcs éco-industriels) ne révèlent pas la dynamique transitionnelle opérant au niveau de la société, ou même de ses sous-parties. La transition est pourtant aussi appelée à ce niveau (CMED, 1987 ; EC, 2019 ; OECD, 2019a, 2019b; UNEP, 2015). Et malgré un constat qui tend à l'unanimité, malgré une préoccupation environnementale qui semble au plus haut, force est de constater que le système socioéconomique dominant se caractérise toujours par le triptyque consacré « extraire - fabriquer - jeter » Les banques marocaines sont parmi les plus importantes de toute l'Afrique et dominent le marché marocain. Les sept plus grandes banques du Maroc représentent $90 \%$ des comptes des dépôts totaux du secteur et $82,3 \%$ des actifs de l'industrie, un secteur qui se modernise progressivement et les banques marocaines commencent également à étendre leur portée en Afrique et dans d'autres parties du monde.

Cette expansion n'a pas toujours été totalement sans heurts ni sans risques, mais elle commence à porter ses fruits. Attijariwafa Bank réalise aujourd'hui environ un tiers de ses revenus hors du Maroc, tandis que les revenus internationaux de la Banque Centrale Populaire ont augmenté de 800\% entre 2009 et 2017. 
Les banques se développent également dans de nouveaux services. En 2017, BTI Bank a lancé une nouvelle offre de banque islamique, donnant accès à des produits et services conformes à la Charia. Cette offre a connu un succès presque sans réserve, non seulement en stimulant l'inclusion financière des citoyens auparavant non bancarisés et en s'étendant à plus de 100 agences, mais aussi en ouvrant de nouveaux marchés pour la BTI Bank.

\section{Figure 2 - De la Renaissance aux Lumières, le renouvellement du rapport à la nature par les sciences expérimentales}

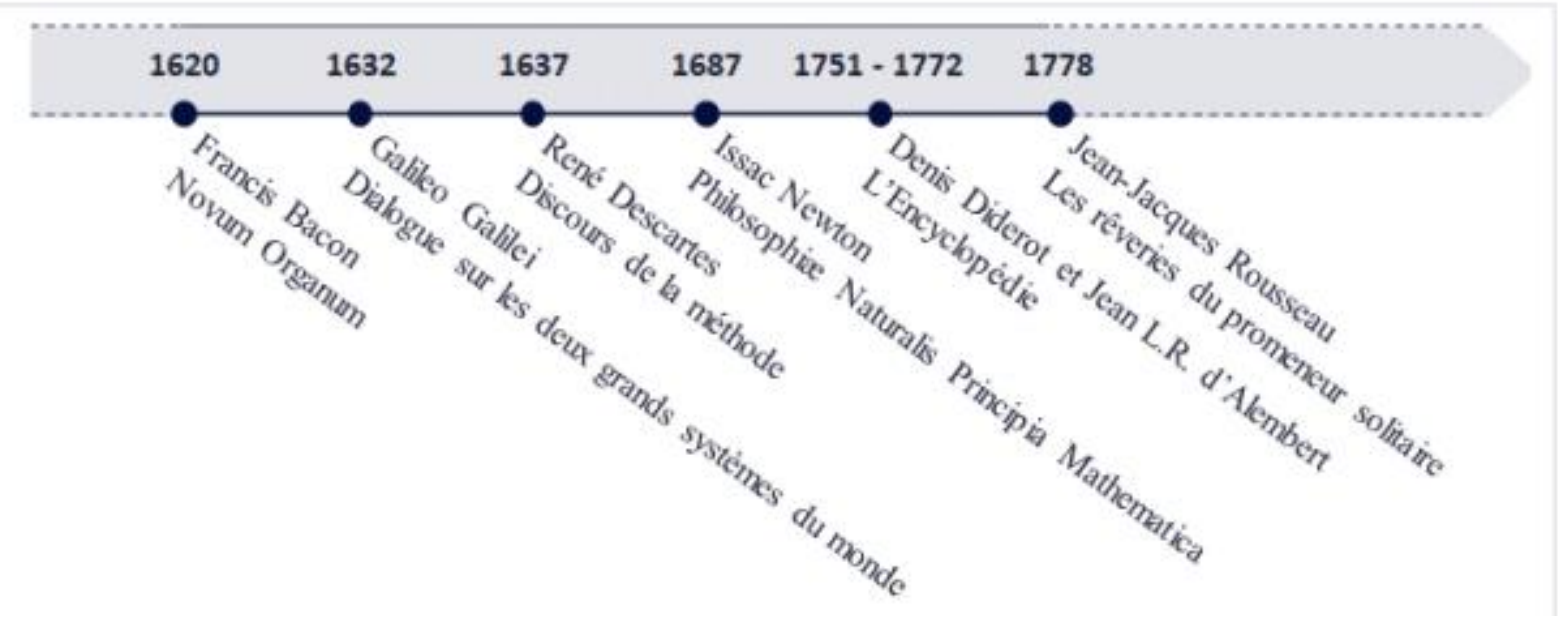

Ce nouveau cadre de pensée bat en brèche la vision religieuse, jusqu'ici prévalente, portée sur la nature. Le monde n'est plus issu de la Création, statique et éternel. La nature cesse d'être perçue comme une expression divine. Elle est dorénavant matière quantifiable et classifiable, mue par une mécanique maitrisable par l'homme. Elle devient un moyen au service de fins humaines, qui lui sont extérieures. Le projet baconien rend explicitement compte de la volonté de domination de la nature par l'homme via le progrès des sciences (Bourg, 1997). L'époque voit alors se développer une vision mécaniste et un nouveau rapport, instrumental, à la nature qui se sont encore accentués sous le siècle des Lumières.

\section{DE LA RENAISSANCE AUX LUMIERES}

Une telle expansion offre des opportunités de croissance significatives pour les banques marocaines, mais une telle stratégie s'accompagne également d'un risque accru. Cela signifie que les fournisseurs de services financiers ont également besoin du bon logiciel de gestion des risques d'entreprise pour se protéger et atténuer ce risque à mesure qu'ils se développent (figure 3). 
Figure 3-Du $19^{\mathrm{eme}}$ siècle à l'avant-première guerre mondiale, la prise de conscience écologique

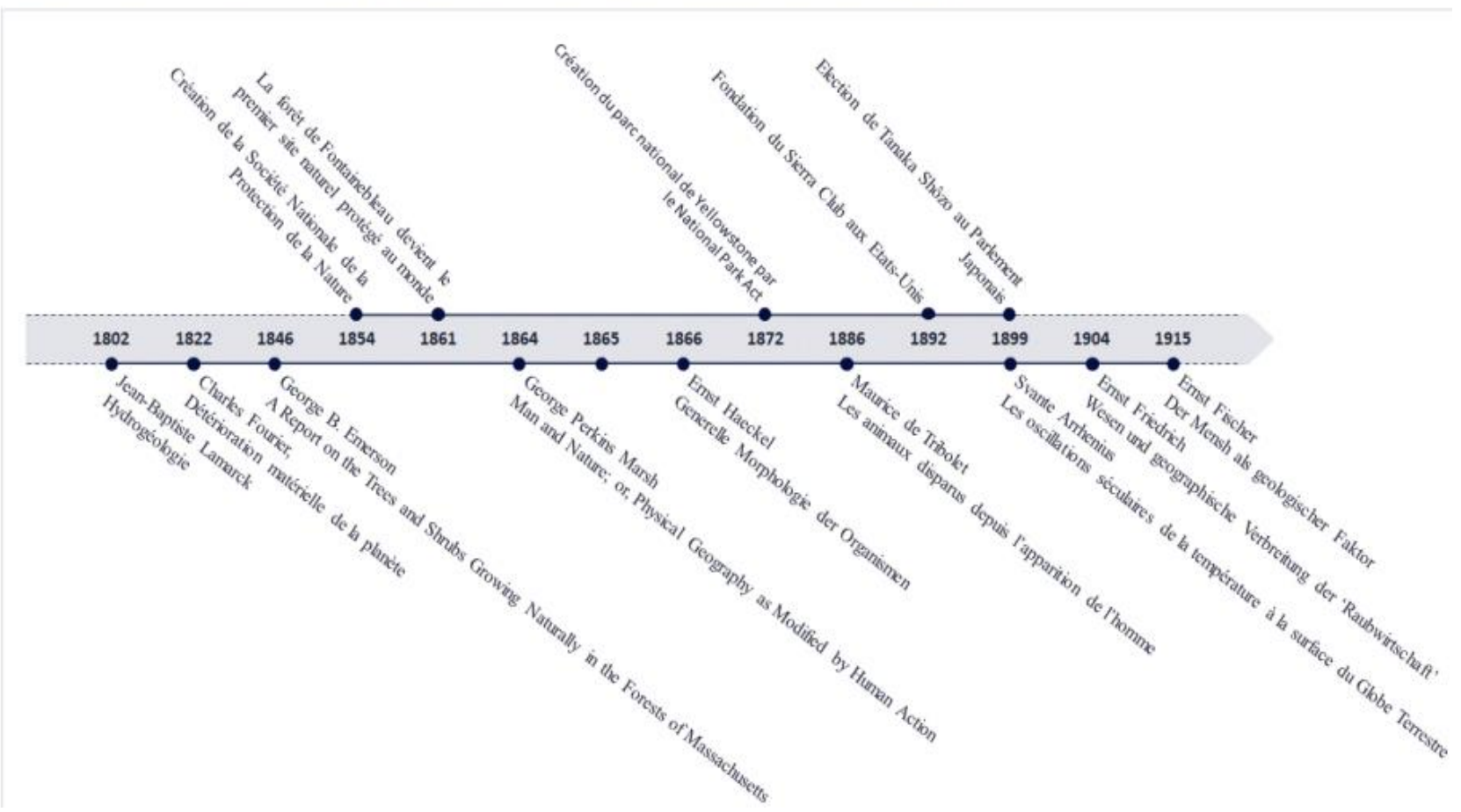

L'acquisition et l'expansion peuvent exercer une pression sur les réserves d'une banque, alors qu'en Afrique de l'Ouest, où l'expansion est importante, certains pays sont politiquement moins stables que d'autres. En raison de la langue, de la culture et des liens historiques, la France est un marché européen naturel pour l'expansion. Mais l'introduction de GDPR en 2018 a signifié une autre couche de conformité réglementaire à gérer. Toutes les entreprises marocaines de services financiers qui détiennent des données sur les citoyens de l'UE doivent se conformer à la GDPR et les pénalités pour non-conformité peuvent signifier certaines des plus grosses amendes que l'industrie a connues.

Une notation Fitch datant de début 2019, mettait en garde contre les risques qu'elle prétendait faire peser sur les banques marocaines. La banque centrale marocaine a réfuté cette affirmation, déclarant qu'elle avait un contrôle réglementaire efficace et effectif du secteur bancaire, contrairement à ce que Fitch suggérait. Fitch est une agence de notation de crédit internationale respectée, et ses avertissements ne peuvent pas être complètement rejetés. 
Figure 4-De l'après seconde guerre mondiale à nos jours, l'écologie comme nouveau courant de pensée

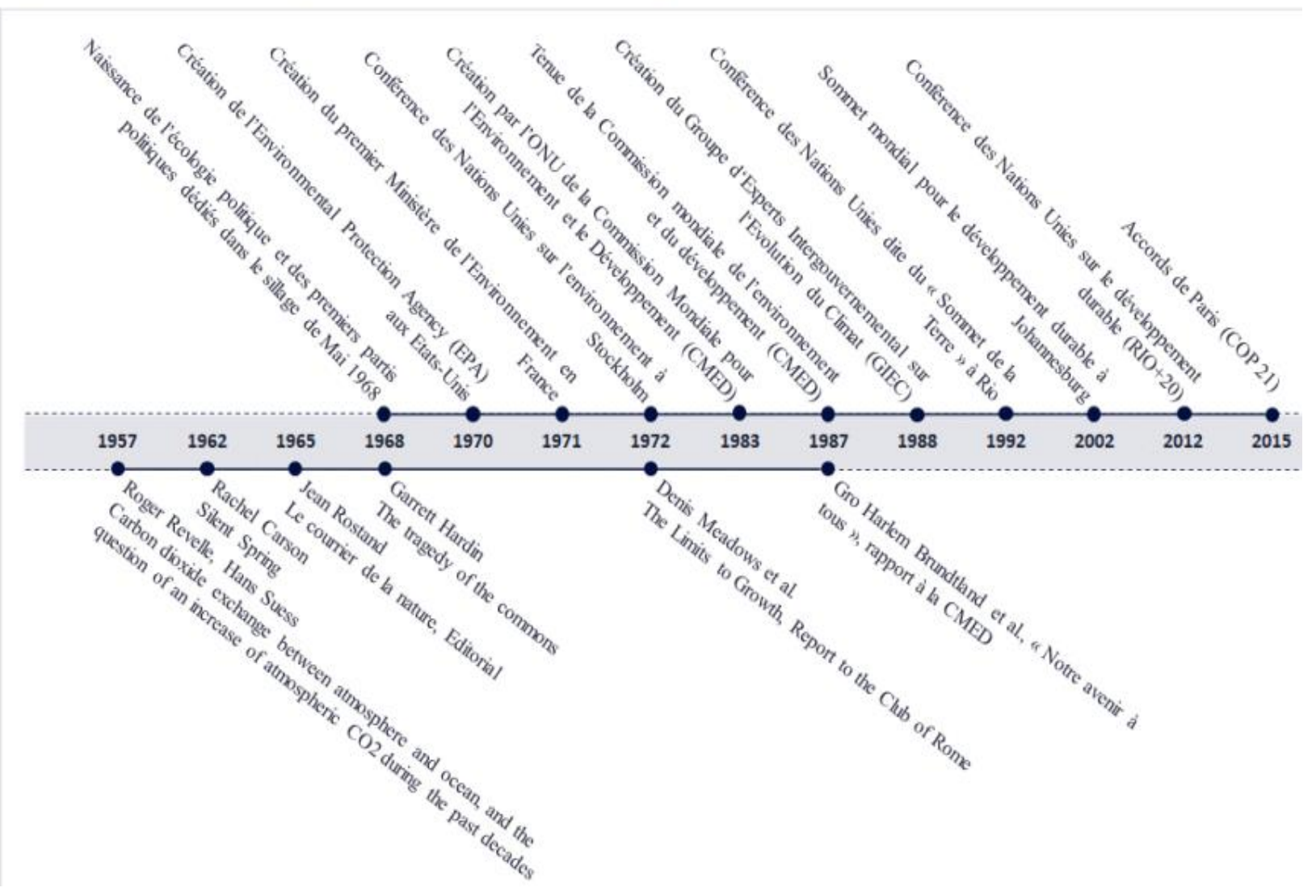

\section{DE L'APRES SECONDE GUERRE MONDIALE A NOS JOURS}

Il existe sans aucun doute des possibilités d'expansion et de croissance des services financiers marocains, mais même si l'on n'est pas d'accord avec la notation de Fitch, il est clair que le secteur a encore un grand besoin de gérer et d'atténuer les risques plus efficacement à mesure qu'il progresse.

Pour ce faire, il faut disposer de la bonne solution logicielle GRC. Il n'est pas nécessaire que ce soit l'outil de GRC le plus cher du marché, mais il doit être adapté aux besoins et aux objectifs globaux de l'organisation concernée. Oxial a récemment lancé ses nouvelles solutions sGRC - sGRC Express et sGRC Suite - qui portent les fonctionnalités GRC à un niveau supérieur. Dans la figure 2 nous présentons la courbe de Farmer. (Figure 2) 
Figure 5 - Projection des consommations de ressources naturelles 2017-2060

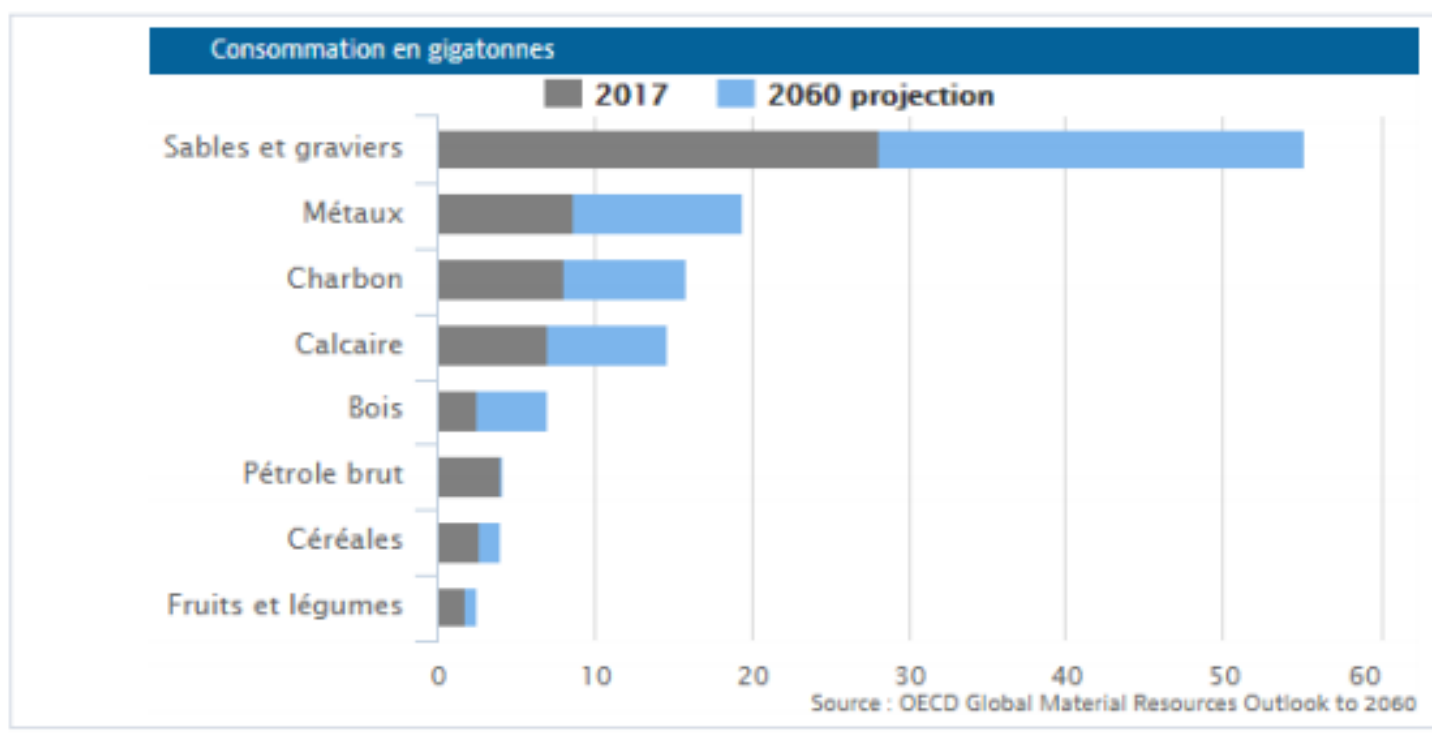

Nous avons intégré la technologie et l'innovation, ainsi que les connaissances, la gouvernance et les processus, la culture et les capacités pour créer une solution qui atténue le risque de manière très efficace et garantit la conformité à toutes les exigences réglementaires. Parce que nos partenaires, nos clients ont accès à certains des consultants les plus compétents en Afrique du Nord, avec une compréhension profonde des services financiers marocains mais aussi du monde entier. Mesure que les entreprises marocaines de services financiers élargissent leur champ d'action, pénètrent de nouveaux marchés et offrent de nouveaux produits et services, il est logique que les risques augmentent également. Si vous êtes une entreprise marocaine de services financiers et que vous souhaitez obtenir de l'aide pour gérer et réduire les risques, veuillez contacter l'un des experts marocains de services financiers ici. 


\section{Figure 6-L'économie circulaire, formalisation de la fondation Ellen MacArthur}

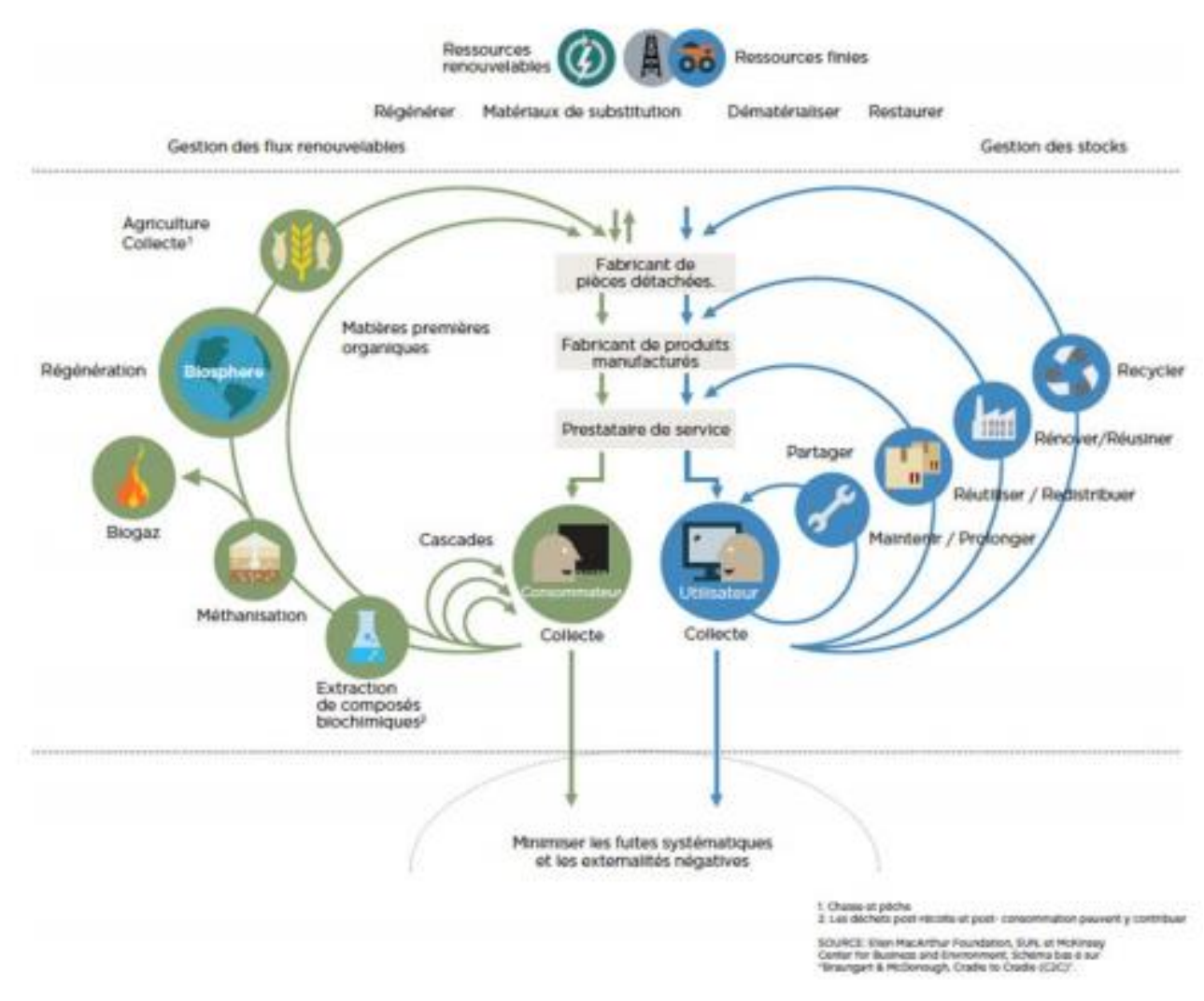

Figure 6 présente l'attaque financière représente une arme de choix pour les entreprises se situant dans un contexte de guerre économique. Les vols de documents et le piratage informatique, tellement craints, figurent loin derrière dans le palmarès des attaques contre les entreprises françaises établi par la section financière de la DCRG, le risque financier représentant plus de $35 \%$ des modalités d'agression. 


\section{Figure 7 - Kalundborg, exemple de symbiose industrielle}

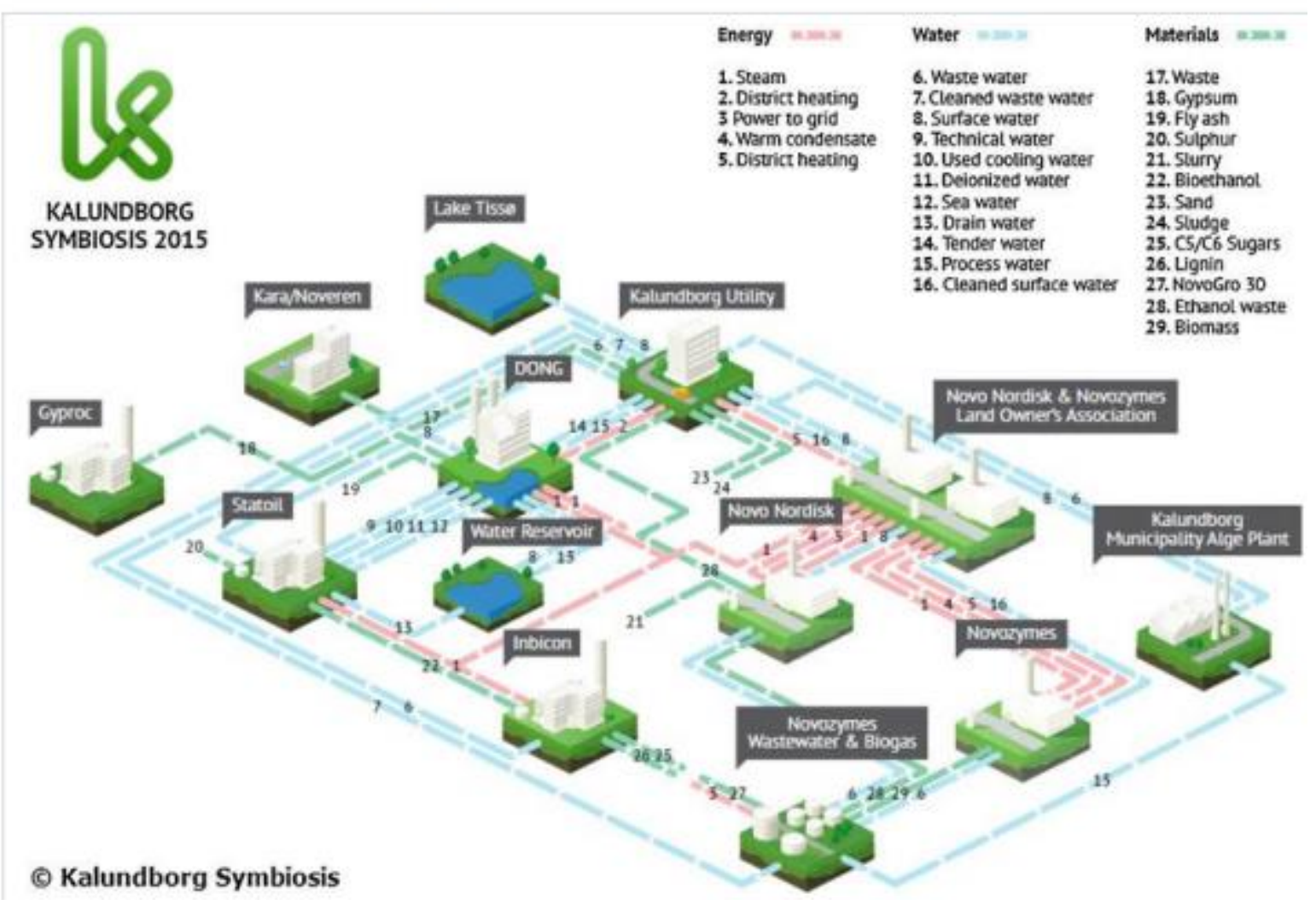

\section{CONCLUSION}

Dans cet article on a met en lien théorie néo-institutionnelle et pratiques relevant de l'économie circulaire. S'appuyant sur plusieurs méthodes inductives, elle s'alimente principalement de publications de presse, de documentation institutionnelle, et d'entretiens menés avec des acteurs de tout type en prise avec l'économie circulaire. Au plan théorique, les résultats permettent de mieux comprendre le rôle des discours dans l'hybridation de pratiques, la dimension institutionnelle de l'économie circulaire, ainsi que l'institutionnalisation de pratiques qui prend appui sur des micro-fondations. Au plan pratique, cette thèse dévoile des ressorts discursifs de diffusion des pratiques relevant de l'économie de fonctionnalité. Elle offre des éléments de diagnostic stratégique permettant d'aborder les transformations du domaine des déchets ménagers en France. Enfin elle décrit les mécanismes de gamification qui engagent le citoyen dans le geste de tri et de réduction des déchets.

\section{REFERENCES}

[1] Rawls, A. W. (2008). Harold Garfinkel, ethnomethodology and workplace studies. Organization Studies, 29, 701-732.

[2] Newton, I. (1687). Principia - Principes mathématiques de la philosophie naturelle (Dunod (août 2011)). Nigam, A., \& Ocasio, W. (2010). Event attention, environmental sensemaking, and change in institutional logics: An inductive analysis of the effects of public attention to Clinton's health care reform initiative. Organization Science, 21, 823-841.

[3] Reay, T., \& Jones, C. (2016). Qualitatively capturing institutional logics. Strategic Organization, 14, 441-454.

[4] Reinert, M. (1983). Une méthode de classification descendante hiérarchique: application à l'analyse lexicale par contexte. Les Cahiers de l'analyse Des Données, 8, 187-198.

[5] Revelle, R., \& Suess, H. E. (1957). Carbon Dioxide Exchange Between Atmosphere and Ocean and the Question of an Increase of Atmospheric CO2 during the Past Decades. Tellus, 9, 18-27. 
[6] Oliver, C. (1992). The antecedents of deinstitutionalization. Organization Studies, 13, 563-588.

[7] ONU. (2017). World Population Prospects: The 2017 Revision, Key Findings and Advance Tables. United Nations, Department of Economic and Social Affairs, Population Divisio.

[8] Pache, A.-C., \& Santos, F. (2010). When worlds collide: The internal dynamics of organizational responses to conflicting institutional demands. Academy of Management Review, 35, 455-476.

[9] ABNAHA, Jamal et ERRAMI, Fadwa. Les Services Financiers au Maroc-Une Industrie Confrontée à des Risques Excessifs. International Journal of Financial Accountability, Economics, Management, and Auditing (IJFAEMA), 2021, vol. 3, no 1, p. 11-16.

[10] NAILI, Said et MORSLI, Amine. Impact of Industrial Atmospheric Emissions on Ambient Air Quality in Arzew Area, Oran State, Algeria. International Journal of Information Technology and Applied Sciences (IJITAS), 2021, vol. 3, no 2, p. 38-42.

[11] ESSAOUDI, MOHAMED et LOTFI, RAJA. Assessment of the Quality of the Training System in Moroccan Higher Education Institutions: Case of the Sciences ans Techniques of Physical and Sports Activities. International Journal of Information Technology and Applied Sciences (IJITAS), 2021, vol. 3, no 2, p. 69-77. 